\title{
REFLEXIVITY ON HILBERT FUNCTION SPACES
}

\author{
A. Iloon Kashkooly ${ }^{1}$, Z. Fattahi ${ }^{2}$ \\ ${ }^{1,2}$ Department of Mathematics \\ University of Yasouj \\ P.O. Box 75918-74831, Yasouj, IRAN
}

\begin{abstract}
In this paper we present sufficient conditions for reflexivity of any powers of the multiplication operator acting on Hilbert spaces of analytic functions on a finitely connected domain. This improves the main result of [21].
\end{abstract}

AMS Subject Classification: 47B37, 47L10

Key Words: reflexive operator, weak operator topology, bounded point evaluation, finitely connected domain, Caratheodory domain

\section{Introduction}

By $H(G)$ and $H^{\infty}(G)$ we will mean respectively the set of analytic functions on a plane domain $G$ and the set of bounded analytic functions on $G$. Assume that $\Omega$ is a finitely connected domain. It is well known that $\Omega$ is conformally equivalent to a circular domain. By a circular domain we mean any domain that is obtained by removing a finite number of disjoint closed subdisks from the open unit disk $\mathbb{D}$. So we let $\Omega=\mathbb{D} \backslash\left(\overline{D_{1}} \cup \ldots \cup \overline{D_{N}}\right)$ where $\bar{D}_{i}=\{z$ : $\left.\left|z-z_{i}\right| \leq r_{i}\right\} \quad(i=1, \ldots, N)$ are disjoint subdisks of the open unit disk $\mathbb{D}$. We can choose $\epsilon_{i}>0(i=1, \ldots, N)$ such that the circles

$$
\Gamma_{i}=\left\{z:\left|z-z_{i}\right|=r_{i}+\epsilon_{i}\right\} \quad(i=1, \ldots, N)
$$

and $\Gamma_{0}=\left\{z:|z|=1-\epsilon_{0}\right\}$ lying in $\Omega$ concentrate to the boundary circles of $\Omega$

Received: $\quad$ May 30, 2016

Revised: December 28, 2016

Published: $\quad$ February 1, 2017

${ }^{\S}$ Correspondence author (c) 2017 Academic Publications, Ltd. url: www.acadpubl.eu 
so that they don't meet each other. We denote $\Omega_{i}=\mathbf{C} \backslash \bar{D}_{i} \quad(i=1, \ldots, N)$. In [3] it is proved that

$$
H^{\infty}(\Omega)=H^{\infty}(\mathbb{D})+H_{0}^{\infty}\left(\Omega_{1}\right)+\ldots+H_{0}^{\infty}\left(\Omega_{N}\right)
$$

where the subscript zero means that the corresponding functions vanish at $\infty$.

Consider a Hilbert space $\mathcal{H}$ of functions analytic on a plane domain $G$, such that for each $\lambda \in G$ the linear functional, $e_{\lambda}$, of evaluation at $\lambda$ is bounded on $\mathcal{H}$. Assume further that $\mathcal{H}$ contains the constant functions and multiplication by the independent variable $z$ defines a bounded linear operator $M_{z}$ on $\mathcal{H}$. The continuity of point evaluations along with the Riesz representation theorem imply that for each $\lambda \in G$ there is a unique function $k_{\lambda} \in \mathcal{H}$ such that $e_{\lambda}(f)=$ $f(\lambda)=<f, k_{\lambda}>, f \in \mathcal{H}$. The function $k_{\lambda}$ is called the reproducing kernel for the point $\lambda$.

A complex valued function $\varphi$ on $G$ for which $\varphi f \in \mathcal{H}$ for every $f \in \mathcal{H}$ is called a multiplier of $\mathcal{H}$ and the collection of all these multipliers is denoted by $\mathcal{M}(\mathcal{H})$. Each multiplier $\varphi$ of $\mathcal{H}$ determines a multiplication operator $M_{\varphi}$ on $\mathcal{H}$ by $M_{\varphi} f=\varphi f, f \in \mathcal{H}$. It is well known that each multiplier is a bounded analytic function on $G$ (see [12]). In fact $\|\varphi\|_{G} \leq\left\|M_{\varphi}\right\|$, where

$$
\|\varphi\|_{G}=\sup \{|\varphi(z)|: z \in G\} .
$$

We shall use the following notation for the norm of the operator $M_{\varphi}$ :

$$
\|\varphi\|_{\infty}=\left\|M_{\varphi}\right\|
$$

We also point out that if $\varphi$ is a multiplier and $\lambda \in G$, then

$$
M_{\varphi}^{*} k_{\lambda}=\overline{\varphi(\lambda)} k_{\lambda}
$$

Recall that if $E$ is a separable Banach space and $A \in B(E)$, then $\operatorname{Lat}(A)$ is by definition the lattice of all invariant subspaces of $A$, and $\operatorname{Alg} \operatorname{Lat}(A)$ is the algebra of all operators $B$ in $B(E)$ such that $\operatorname{Lat}(A) \subset \operatorname{Lat}(B)$. For the algebra $B(E)$, the weak operator topology is the one induced by the family of seminorms $p_{x^{*}, x}(A)=\left|<A x, x^{*}\right\rangle \mid$ where $x \in E, x^{*} \in E^{*}$ and $A \in B(E)$. Hence $A_{\alpha} \longrightarrow A$ in the weak operator topology if and only if $A_{\alpha} x \longrightarrow A x$ weakly. Also similarly $A_{\alpha} \longrightarrow A$ in the strong operator topology if and only if $A_{\alpha} x \longrightarrow A x$ in the norm topology. An operator $A$ in $B(E)$ is said to be reflexive if $\operatorname{AlgLat}(A)=W(A)$, where $W(A)$ is the smallest subalgebra of $B(E)$ that contains $A$ and the identity $I$ and is closed in the weak operator topology. 


\section{Main Results}

The operator $M_{z}$ has been the focus of attention for several decades and many of its properties have been studied (e.g. [2,12]). In [1o] Sarason proved that normal operators are reflexive. It was shown by J. Deddens (see [4]) that every isometry is reflexive. Also, R. Olin and J. Thomson (see [8]) have shown that subnormal operators are reflexive. H. Bercovici, C. Foias, J. Langsam, and C. Pearcy (see [1]) have shown that (BCP)-operators are reflexive. The reflexive operators on a finite dimensional space were characterized by J. Deddens and P. A. Fillmore (see [5]). In [7,11,14 - 21] some sufficient conditions for the reflexivity of multiplication operators on some function spaces have been investigated. Also, reflexivity of canonical models were studied in [6]. In [17] it is proved that if $M_{z}$ is invertible on the space $L^{p}(\beta)$, then it is reflexive. In this article we would like to give some sufficient conditions so that the powers of the operator $M_{z}$, acting on a Hilbert space of analytic functions on a finitely connected domain, becomes reflexive. This extends the main result of the paper [21]. For a good source of reflexivity see [9].

From now on, let $\Omega$ be a finitely connected domain in the complex plane and suppose that the Hilbert space $\mathcal{H}$ under consideration satisfy the following axioms:

Axiom 1. $\mathcal{H}$ is a subspace of the space of all analytic functions on $\Omega$.

Axiom 2. For each $\lambda \in \Omega$, the linear functional of evaluation at $\lambda, e(\lambda)$, is bounded on $\mathcal{H}$.

Axiom 3. The sequence $\left\{f_{k}\right\}_{k}$ is an orthogonal basis for $\mathcal{H}$ where $f_{k}(z)=$ $z^{k}$ for all integers $k$.

For $h \in H(\mathbb{D}) \cap \mathcal{M}(\mathcal{H})$ and $w \in \partial \mathbf{D}$, define $h_{w}$ by $h_{w}(z)=h(w z)$. Thus $\hat{h}_{w}(n)=w^{n} \hat{h}(n)$ for all $n$. Note that since $|w|=1$, we have

$$
\left\|h_{w}\right\|^{2}=\sum_{n}\left|\hat{h}_{w}(n)\right|^{2}\left\|f_{n}\right\|^{2}=\sum_{n}|\hat{h}(n)|^{2}\left\|f_{n}\right\|^{2}=\|h\|^{2} .
$$

Also, we say that $H(\mathbb{D}) \cap \mathcal{M}(\mathcal{H})$ is isometrically rotation invariant if whenever $\varphi \in H(\mathbb{D}) \cap \mathcal{M}(\mathcal{H})$, then $\varphi_{e^{-i \theta}} \in H(\mathbb{D}) \cap \mathcal{M}(\mathcal{H})$ and $\|\varphi\|_{\infty}=\left\|\varphi_{e^{-i \theta}}\right\|_{\infty}$ for all $\theta \in \mathbb{R}$.

Furthermore, we assume that $\mathcal{H}$ holds in the following axiom:

Axiom 4. $H(\mathbb{D}) \cap \mathcal{M}(\mathcal{H})$ is isometrically rotation invariant.

For the proof of the main result we will need the following lemmas. 
Lemma 2.1. Let $\varphi \in H(\mathbb{D}) \cap \mathcal{M}(\mathcal{H})$. Then:

(i) If $w \longrightarrow 1$, then $M_{\varphi_{w}} \longrightarrow M_{\varphi}$ in the strong operator topology.

(ii) If $g$ is a continuous complex valued function on $\partial \mathbf{D}$ and $d \lambda=|d w| / 2 \pi$ is the normalized Lebesgue measure on $\partial \mathbf{D}$, then the operator

$$
\int_{\partial \mathbf{D}} \varphi_{w} g(w) d \lambda
$$

defined by

$$
\left(\int_{\partial \mathbb{D}} \varphi_{w} g(w) d \lambda\right) f=\int_{\partial \mathbb{D}} g(w) M_{\varphi_{w}} f d \lambda
$$

is in $\mathcal{M}(\mathcal{H})$ and

$$
\left\|\int_{\partial \mathbf{D}} \varphi_{w} g(w) d \lambda\right\|_{\infty} \leq\left\|M_{\varphi}\right\| \int_{\partial \mathbf{D}}|g| d \lambda .
$$

Proof. (i) For $w \in \partial \mathbf{D}$ we have

$$
\begin{aligned}
\left\|\left(M_{\varphi_{w}}-M_{\varphi}\right) f_{m}\right\|^{2} & =\left\|\left(\varphi_{w}-\varphi\right) f_{m}\right\|^{2}=\left\|\sum_{n}\left(\hat{\varphi}_{w}(n)-\hat{\varphi}(n)\right) f_{n+m}\right\|^{2} \\
& =\sum_{n}\left|\hat{\varphi}_{w}(n)-\hat{\varphi}(n)\right|^{2}\left\|f_{n+m}\right\|^{2} \\
& =\sum_{n}|\hat{\varphi}(n)|^{2}\left|w^{n}-1\right|\left\|f_{n+m}\right\|^{2} .
\end{aligned}
$$

Thus for all $m, M_{\varphi_{w}} f_{m} \longrightarrow M_{\varphi} f_{m}$ as $w \longrightarrow 1$. Since $\left\|M_{\varphi_{w}}\right\|=\left\|M_{\varphi}\right\|<\infty$ for all $w \in \partial \mathbf{D}$, indeed $M_{\varphi_{w}} \longrightarrow M_{\varphi}$ in the strong operator topology.

(ii) First note that the strong operator continuity of $\varphi_{w}$ allows us to define

$$
\int_{\partial \mathbf{D}} \varphi_{w} g(w) f d \lambda
$$

for all $f \in \mathcal{H}$. If $f, h \in \mathcal{H}$, then

$$
<\int_{\partial \mathbf{D}} \varphi_{w} g(w) f d \lambda, h>=\int_{\partial \mathbf{D}} g(w)<\varphi_{w} f, h>d \lambda
$$

Since $\left\|M_{\varphi}\right\|=\left\|M_{\varphi_{w}}\right\|$, we get

$$
\left\|\int_{\partial \mathbb{D}} \varphi_{w} g(w) f d \lambda\right\| \leq\left\|M_{\varphi}\right\|\|f\| \int_{\partial \mathbb{D}}|g| d \lambda .
$$


Hence

$$
\left\|\left(\int_{\partial \mathbf{D}} \varphi_{w} g(w) d \lambda\right) f\right\|=\left\|\int_{\partial \mathbf{D}} g(w) M_{\varphi_{w}} f d \lambda\right\| \leq\left\|M_{\varphi}\right\|\|f\| \int_{\partial \mathbf{D}}|g| d \lambda .
$$

This completes the proof.

Throughout this paper we suppose that $M_{z}$ is bounded on $\mathcal{H}$. In the following by $H(G)$ and $H^{\infty}(G)$ we will mean respectively the set of analytic functions on a plane domain $G$ and the set of bounded analytic functions on $G$.

Lemma 2.2. Let $\varphi \in H(\mathbb{D}) \cap \mathcal{M}(\mathcal{H})$ and let $p$ be a polynomial. Then $\varphi * p \in \mathcal{M}(\mathcal{H})$ and

$$
\int_{\partial \mathbb{D}} \varphi_{w} p(\bar{w}) d \lambda=M_{\varphi * p}
$$

where

$$
(\varphi * p)(z)=\sum_{i} \hat{\varphi}(i) \hat{p}(i) f_{i}
$$

Proof. It is enough to consider the case $p=f_{j}$. Define the operator $L$ by

$$
L=\int_{\partial \mathbf{D}} \varphi_{w} f_{j}(\bar{w}) d \lambda .
$$

We should prove that the operators $L$ and $M_{\hat{\varphi}(j) f_{j}}$ have the same matrix entries with respect to the orthogonal basis $\left\{f_{j}\right\}_{j}$. We have

$$
<M_{\hat{\varphi}(j) f_{j}} f_{m}, f_{n}>=\hat{\varphi}(j)<f_{m+j}, f_{n}>
$$

which is equal to $\hat{\varphi}(n-m)\left\|f_{n}\right\|^{2}$ whenever $n=m+j$, and is 0 else. On the other hand we note that

$$
\begin{aligned}
<L f_{m}, f_{n}> & =\int_{\partial \mathbb{D}} \bar{w}^{j}<\varphi_{w} f_{m}, f_{n}>d \lambda \\
& =\int_{\partial \mathbb{D}} \bar{w}^{j} \hat{\varphi}_{w}(n-m)\left\|f_{n}\right\|^{2} d \lambda \\
& =\int_{\partial \mathbb{D}} \bar{w}^{j} w^{n-m} \hat{\varphi}(n-m)\left\|f_{n}\right\|^{2} d \lambda \\
& =\hat{\varphi}(n-m)\left\|f_{n}\right\|^{2} \int_{\partial \mathbf{D}} w^{n-m-j} d \lambda
\end{aligned}
$$

which is equal to $\hat{\varphi}(n-m)\left\|f_{n}\right\|^{2}$ whenever $n=m+j$, and is 0 else. Hence $L=M_{\hat{\varphi}(j) f_{j}}$. Since $\left\{f_{j}\right\}_{j}$ is a basis for $\mathcal{H}$, the proof is complete. 
Lemma 2.3. If $\varphi \in H(\mathbb{D}) \cap \mathcal{M}(\mathcal{H})$, then for the sequence of polynomials $\left\{r_{n}\right\}$ where $\hat{r_{n}}(j)=\left(1-\frac{j}{n+1}\right) \hat{\varphi}(j)$ whenever $j=0, \ldots, n$ and is 0 else, we have $M_{r_{n}} \rightarrow M_{\varphi}$ in the weak operator topology.

Proof. Let $\varphi \in H(\mathbb{D}) \cap \mathcal{M}(\mathcal{H})$. Since $\mathbb{D}$ is a Caratheodory domain, $\varphi$ can be represenyed by the power series $\sum_{k=0}^{\infty} \hat{\varphi}(k) z^{k}$. Put

$$
P_{n}(\varphi)=\sum_{k=0}^{n}\left(1-\frac{k}{n+1}\right) \hat{\varphi}(k) z^{k}, \quad n \geq 0
$$

and

$$
K_{n}(w)=\sum_{|k| \leq n}\left(1-\frac{|k|}{n+1}\right) w^{k}, \quad w \in \partial U, \quad n \geq 0
$$

Then

$$
\int_{\partial \mathbf{D}} \varphi_{w} K_{n}(\bar{w}) d \lambda=M_{\varphi * K_{n}}, \quad n \geq 0
$$

where

$$
\left(\varphi * K_{n}\right)(z)=\sum_{j=0}^{n} \hat{\varphi}(j) \hat{K}_{n}(j) z^{j}=P_{n}(\varphi) .
$$

Note that $K_{n} \geq 0$ and

$$
\int_{\partial \mathbf{D}} K_{n} d \lambda=1
$$

For all $n \geq 0, P_{n}(\varphi) \in H(\mathbb{D}) \cap \mathcal{M}(\mathcal{H})$ and by Lemma 2.1 (ii), we get

$$
\left\|M_{P_{n}(\varphi)}\right\|=\left\|M_{\varphi * K_{n}}\right\| \leq\left\|M_{\varphi}\right\| \int_{\partial \mathbf{D}} K_{n} d \lambda=\left\|M_{\varphi}\right\| .
$$

Put $r_{n}=P_{n}(\varphi)$ and note that $M_{r_{n}}$ is represented by the matrix whose $(\mathrm{i}, \mathrm{j})$-th entry is

$$
<M_{r_{n}} f_{j}, f_{i}>=\hat{r}_{n}(i-j)\left\|f_{i}\right\|^{2}=\left(1-\frac{i-j}{n}\right) \hat{\varphi}(i-j)\left\|f_{i}\right\|^{2}
$$

Hence

$$
\lim _{n}<M_{r_{n}} f_{j}, f_{i}>=<M_{\varphi} f_{j}, f_{i}>
$$

for all base elements $f_{j}$ and $f_{i}$ in $\mathcal{H}$. By the boundedness of the sequence $\left\{M_{r_{n}}\right\}$, we have $M_{r_{n}} \rightarrow M_{\varphi}$ in the weak operator topology. This completes the proof. 
Corollary 2.4. If $\varphi \in H(\mathbb{D}) \cap \mathcal{M}(\mathcal{H})$, then $M_{\varphi} \in W\left(M_{z}\right)$.

Proof. Let $\varphi \in H(\mathbb{D}) \cap \mathcal{M}(\mathcal{H})$. Then by the proof of Lemma 2.3, we get $M_{r_{n}} \rightarrow M_{\varphi}$ in the weak operator topology where $r_{n}=P_{n}(\varphi)$. Since $r_{n}$ is a polynomial and $M_{r_{n}}=r_{n}\left(M_{z}\right)$, we conclude that $M_{\varphi} \in W\left(M_{z}\right)$.

Theorem 2.5. For all $k \geq 1$, the operator $M_{z^{k}}$ is reflexive on $\mathcal{H}$.

Proof. $\Omega=\mathbb{D} \backslash\left(\bar{D}_{1} \cup \ldots \cup \overline{D_{N}}\right)$ where $\bar{D}_{i}=\left\{z:\left|z-z_{i}\right| \leq r_{i}\right\} \quad(i=1, \ldots, N)$ are disjoint subdisks of the open unit disk $\mathbb{D}$. Choose $\epsilon_{i}>0(i=1, \ldots, N)$ such that the circles

$$
\Gamma_{i}=\left\{z:\left|z-z_{i}\right|=r_{i}+\epsilon_{i}\right\} \quad(i=1, \ldots, N)
$$

and $\Gamma_{0}=\left\{z:|z|=1-\epsilon_{0}\right\}$ lying in $\Omega$ concentrate to the boundary circles of $\Omega$ so that they don't meet each other. Denote $\Omega_{i}=\mathbf{C} \backslash \bar{D}_{i} \quad(i=1, \ldots, N)$.

First, we note that convergence in $\mathcal{H}$ implies uniform convergence on compact subsets of $\Omega$. For this let $K$ be a compact subset of $\Omega$ and consider the family of bounded linear functionals $\left\{e_{\lambda}: \lambda \in K\right\}$. If $f \in \mathcal{H}$, then $\|f\|_{K}<\infty$. So by the Principle of Uniform Boundedness Theorem, the family $\left\{e_{\lambda}: \lambda \in K\right\}$ is bounded. Put $c=\sup \left\{\left\|e_{\lambda}\right\|: \lambda \in K\right\}$ and let a sequence $\left\{f_{n}\right\}_{n}$ converges to $f$ in $\mathcal{H}$. Then we have

$$
\left|f_{n}(\lambda)-f(\lambda)\right| \leq\left\|e_{\lambda}\right\|\left\|f_{n}-f\right\| \leq c\left\|f_{n}-f\right\|
$$

Hence convergence in $\mathcal{H}$ implies uniform convergence on compact subsets of $\Omega$. Set

$$
L_{0}=\left\{f \in \mathcal{H}: \int_{\Gamma_{0}} z^{n} f(z) d z=0, \quad n=0,1,2, \cdots\right\} .
$$

Note that $L_{0}$ is a subspace of $\mathcal{H}$. To see that $L_{0}$ is closed, let $\left\{g_{k}\right\}$ be a sequence in $L_{0}$ such that $g_{k} \rightarrow h$ in $\mathcal{H}$. Since $\Gamma_{0}$ is a compact subset of $\Omega$, it is now easy to see that $h \in L_{0}$ and so $L_{0}$ is closed in $\mathcal{H}$. Also, clearly $L_{0}$ is invariant under $M_{z}$ and contains the constants. Let $k \in \mathbb{N}$ and note that $W\left(M_{z^{k}}\right) \subset \operatorname{AlgLat}\left(M_{z^{k}}\right)$. On the other hand, let $A \in \operatorname{AlgLat}\left(M_{z^{k}}\right)$. Since $\operatorname{Lat}\left(M_{z}\right) \subset \operatorname{Lat}\left(M_{z^{k}}\right)$, thus we have $\operatorname{Lat}\left(M_{z}\right) \subset \operatorname{Lat}(A)$. This implies that $A \in \operatorname{AlgLat}\left(M_{z}\right)$. Note that since $M_{z}^{*} e(\lambda)=\bar{\lambda} e(\lambda)$ for all $\lambda$ in $\Omega$, the one dimensional span of $e(\lambda)$ is invariant under $M_{z}^{*}$. Therefore it is invariant under $A^{*}$ and we can write $A^{*} e(\lambda)=\overline{\varphi(\lambda)} e(\lambda), \lambda \in \Omega$. So

$$
<A f, e(\lambda)>=<f, A^{*} e(\lambda)>=\varphi(\lambda) f(\lambda)
$$


for all $f \in \mathcal{H}$ and $\lambda \in \Omega$. This implies that $A=M_{\varphi}$ and $\varphi \in \mathcal{M}(\mathcal{H})$, hence $\varphi \in H^{\infty}(\Omega)$. Since $L_{0} \in \operatorname{Lat}\left(M_{z}\right)$, we have $A L_{0} \subset L_{0}$, so $A 1=\varphi \in L_{0}$. By applying the Cauchy integral formula we can write $\varphi=\varphi_{0}+\varphi_{1}+\ldots+\varphi_{N}$ where $\varphi_{0} \in H(\mathbb{D})$ and $\varphi_{i} \in H_{0}\left(\Omega_{i}\right)(\mathrm{i}=1, \ldots, \mathrm{N})$ (here $H_{0}\left(\Omega_{i}\right)$ denotes the space of all functions in $H\left(\Omega_{i}\right)$ that vanishes at $\left.\infty\right)$. Set $g=\varphi_{1}+\ldots+\varphi_{N}$. Therefore $g$ is analytic in $\operatorname{ext}\left(\Gamma_{0}^{\prime}\right)$ the unbounded component of $\mathbf{C} \backslash \Gamma_{0}^{\prime}$ where the circle $\Gamma_{0}^{\prime}$ is chosen sufficiently close to $\Gamma_{0}$ with smaller radius so that $\Gamma_{0}$ lies in $\operatorname{ext}\left(\Gamma_{0}^{\prime}\right)$. Now, we can write

$$
g(z)=\sum_{n=-\infty}^{-1} \hat{g}(n) z^{n}, \quad z \in \operatorname{ext}\left(\Gamma_{0}^{\prime}\right) .
$$

Note that

$$
\hat{g}(n)=\frac{1}{2 \pi i} \int_{\Gamma_{0}} g(z) z^{-(n+1)} d z, \quad n<0 .
$$

Since $\varphi_{0} \in H(\mathbb{D})$, we have

$$
\int_{\Gamma_{0}} z^{n} \varphi_{0}(z) d z=0, \quad n=0,1,, 2, \cdots .
$$

But $\varphi \in L_{0}$, thus we get

$$
\int_{\Gamma_{0}} z^{n} g(z) d z=0, \quad n=0,1,2, \cdots
$$

From this it follows that $\hat{g}(n)=0$ for all integers $n \leq-1$ and so $g(z)=0$, $z \in \operatorname{ext}\left(\Gamma_{0}^{\prime}\right)$. Hence $g \equiv 0$ which implies that $\varphi=\varphi_{0} \in H(\mathbb{D})$. Thus $\varphi \in$ $H(\mathbb{D}) \cap \mathcal{M}(\mathcal{H})$ and so by Lemma 2.3, there exists a sequence of polynomials $\left\{r_{n}\right\}$ such that $M_{r_{n}} \rightarrow M_{\varphi}$ in the weak operator topology. Now, we use a similar method used in the proof of the main theorem in [21]: let $\mathcal{M}_{k}$ be the closed linear span of the set $\left\{f_{n k}: n \geq 0\right\}$ (recall that $f_{i}(z)=z^{i}$ for all $i$ ). We have $M_{z^{k}} f_{n k}=f_{(n+1) k} \in \mathcal{M}_{k}$ for all $n \geq 0$. Thus $\mathcal{M}_{k} \in \operatorname{Lat}\left(M_{z^{k}}\right)$, and so $\mathcal{M}_{k} \in \operatorname{Lat}\left(M_{\varphi}\right)$. Let $\varphi(z)=\sum_{n=0}^{\infty} \hat{\varphi}(n) z^{n}$. Since $1 \in \mathcal{M}_{k}$, thus $M_{\varphi} 1=\varphi \in \mathcal{M}_{k}$. Hence $\hat{\varphi}(i)=0$ for all $i \neq n k, n \geq 0$. Now, by a consequence of the particular construction of $r_{n}$ used in Lemma 2.3, each $r_{n}$ should be a polynomial in $z^{k}$, i.e., $r_{n}(z)=q_{n}\left(z^{k}\right)$ for some polynomial $q_{n}$. Thus $M_{r_{n}}=r_{n}\left(M_{z}\right)=q_{n}\left(M_{z^{k}}\right) \rightarrow A$ in the weak operator topology. Hence $A \in W\left(M_{z^{k}}\right)$. Thus $M_{z^{k}}$ is reflexive and so the proof is complete.

In the following we give an example of a Hilbert space for which the axioms 1 through 4, hold. 
Example 2.6. Let $\{\beta(n)\}_{n=-\infty}^{\infty}$ be a sequence of positive numbers satisfying $\beta(0)=1$. The space $L^{2}(\beta)$ consists of all formal Laurent series $f(z)=$ $\sum_{n=-\infty}^{\infty} \hat{f}(n) z^{n}$ such that the norm

$$
\|f\|=\|f\|_{\beta}=\left(\sum_{n=-\infty}^{\infty}|\hat{f}(n)|^{2} \beta(n)^{2}\right)^{\frac{1}{2}}
$$

is finite. These are Hilbert spaces with the norm $\|\cdot\|_{\beta}$, see [13]. Let $\hat{f}_{k}(n)=$ $\delta_{k}(n)$, so $f_{k}(z)=z^{k}$ and $\left\|f_{k}\right\|=\beta(k)$. Let $M_{z}$ be bounded on $L^{2}(\beta)$ and consider the following notations:

$$
\begin{aligned}
& r_{0}=\varlimsup \lim \beta(-n)^{\frac{-1}{n}} \quad ; \quad \Omega_{0}=\left\{z \in \mathbf{C}:|z|>r_{0}\right\} \\
& r_{1}=\underline{\lim \beta(n)^{\frac{1}{n}}} ; \Omega_{1}=\left\{z \in \mathbf{C}:|z|<r_{1}\right\} \\
& \Omega=\Omega_{0} \cap \Omega_{1}=\left\{z \in \mathbb{C}: r_{0}<|z|<r_{1}\right\} .
\end{aligned}
$$

Assume that $r_{0}<r_{1}=1$. Then, $\Omega$ is clearly a circular domain and $L^{2}(\beta) \subset$ $H(\Omega)$ (see [13, Theorem 10'(ii), page 79]), so Axiom 1 holds. Also, each point of $\Omega$ is a bounded point evaluation on $L^{2}(\beta)$ (see $\left[13\right.$, Theorem $10^{\prime}$ (ii), page 79 ), so Axiom 2 holds. By proposition 6 in [13, page 57], $\left\{f_{k}\right\}_{k \in \boldsymbol{Z}}$ is an orthogonal basis for $L^{2}(\beta)$ and so Axiom 3 holds. Furthermore, Axiom 4 holds by Proposition 28 in [13, page 88$]$.

Corollary 2.7. For all $k \geq 1$, the operator $M_{z^{k}}$ is reflexive on $L^{2}(\beta)$.

Corollary 2.8. Let $M_{z}$ be invertible on $L^{2}(\beta)$. Then $M_{z^{k}}$ is reflexive for all integers $k$.

Proof. By Corollary 2.7, $M_{z^{k}}$ is reflexive for all positive integers $k$. Note that since $M_{z} f_{m}=f_{m+1}$, we have $M_{z}^{-1} f_{m}=f_{m-1}$ for all $m$. Let $f_{m}^{\prime}=f_{-m}$. Then $M_{z}^{-1} f_{m}^{\prime}=f_{m+1}^{\prime}$ for all $m$. So $\left\{f_{m}^{\prime}\right\}$ is shifted (forward) by $M_{z}^{-1}$. Hence $M_{z}^{-k}$ is reflexive for all $k \geq 1$. But the identity operator is also reflexive, thus indeed $M_{z^{k}}$ is reflexive for all integers $k$.

Remark 2.9. Theorem 2.5 can be extended by the same proof to a Banach space setting instead of a Hilbert space and so the main result of [21] is clearly obtained for the Banach spaces $L^{p}(\beta)$ where $1<p<\infty$. 


\section{References}

[1] H. Bercovici, C. Foias, J. Langsam, and C. Pearcy, (BCP)- operators are reflexive, Mich. Math. J., 29 (1982), 371-379.

[2] P.S. Bourdon and J.H. Shapiro, Spectral and common cyclic vectors, Michigan Math. J., 37 (1990), 71-90.

[3] B. Chevereau, C. Pearcy, and A. Shields, Finitely connected domains $G$, representations of $H^{\infty}(G)$, and invariant subspaces, J. Operator Theory, 6 (1981), 375-405.

[4] J. A. Deddens, Every isometry is reflexive, Proc. Amer. Math. Soc., 28 (1971), 509-512.

[5] J. A. Deddens and P. A. Fillmore, Reflexive linear transformations, Linear Algebra and Appl., 10 (1975), 89-93.

[6] Y. N. Dehghan and B. Yousefi, Reflexivity of canonical models associated with generalized Bergman kernels, Korean Annals of Math., 22, No. 1 (2005), 95-102.

[7] M. Faghih Ahmadi and K. Hedayatian, On the reflexivity of hyponormal and weighted shift operators, Acta Mathematica Scientia, 30B, No. 4 (2010), 1100-1104.

[8] R. Olin and J. Thomson, Algebras of subnormal operators, J. Functional Anal., 37 (1980), 271-301.

[9] H. Radjavi and P. Rosenthal, Invariant Subspaces, Springer-Verlag, New York, 1971.

[10] D. Sarason, Invariant subspaces and unstarred operator algebras, Pacific J. Math., 17 (1966), 511-517.

[11] K. Seddighi and B. Yousefi, On the reflexivity of operators on function spaces, Proc. Amer. Math. Soc., 116 (1992), 45-52.

[12] A. Shields and L. Wallen, The commutants of certain Hilbert space operators, Ind. Univ. Math. J., 20 (1971), 777-788.

[13] A. L. Shields, Weighted shift operators and analytic functions theory, Math. Surveys, A.M.S. Providence, 13 (1974), 49-128.

[14] B. Yousefi, K. Seddighi and K. Hedayatian, On reflexivity of the multiplication operators on Dirichlet spaces, Math. Japonica, 38 (1993), 1189-1194.

[15] B. Yousefi, Multiplication operators on Hilbert spaces of analytic functions, Archiv der Mathematik, 83, No. 6 (2004), 536-539.

[16] B. Yousefi and Y.N. Dehghan, Reflexivity on weighted Hardy spaces, Southeast Asian Bulletin of Mathematics, 28 (2004), 587-593.

[17] B. Yousefi, On the eighteenth question of Allen Shields, International Journal of Mathematics, 16, No. 1 (2005), 1-6.

[18] B. Yousefi and S. Jahedi, Reflexivity of the multiplication operator on the weighted Hardy spaces, Southeast Asian Bulletin of Mathematics, 31 (2007), 163-168.

[19] B. Yousefi and J. Doroodgar, Reflexivity on Banach spaces of analytic functions, Journal of Mathematical Extension, 3, No. 1 (2009), 87-93.

[20] B. Yousefi and A. Khaksari, Multiplication operators on analytic functional spaces, Taiwanese Journal of Mathematics, 13, No. 4 (2009), 1159-1165.

[21] B. Yousefi and Sh. Khoshdel, Reflexivity of powers of the multiplication operator on special function spaces, Acta Mathematica Scientia, 32B, No. 6 (2012), 2279-2284. 